Please do not remove this page

RMIT

UNIVERSITY

\title{
Inducing tunable switching behavior in a single memristor
}

Ahmed, Taimur; Walia, Sumeet; Mayes, Edwin; Ramanathan, Rajesh; Guagliardo, Paul; Bansal, Vipul; Bhaskaran, Madhu

https://researchrepository.rmit.edu.au/esploro/outputs/9921863159601341/filesAndLinks?institution=61RMIT_INST\&index=null

Ahmed, T., Walia, S., Mayes, E., Ramanathan, R., Guagliardo, P., Bansal, V., Bhaskaran, M., Yang, J., \& Sriram, S. (2018). Inducing tunable switching behavior in a single memristor. Applied Materials Today, 11, 280-290. https://doi.org/10.1016/j.apmt.2018.03.003

Document Version: Accepted Manuscript

Published Version: https://doi.org/10.1016/j.apmt.2018.03.003

Repository homepage: https://researchrepository.rmit.edu.au

(c) 2018 Published by Elsevier Ltd.

Downloaded On 2023/04/26 18:38:02 +1000

Please do not remove this page 
Thank you for downloading this document from the RMIT Research Repository.

The RMIT Research Repository is an open access database showcasing the research outputs of RMIT University researchers.

RMIT Research Repository: http://researchbank.rmit.edu.au/

\section{Citation:}

Ahmed, T, Walia, S, Mayes, E, Ramanathan, R, Guagliardo, P, Bansal, V, Bhaskaran, M, Yang, J and Sriram, S 2018, 'Inducing tunable switching behavior in a single memristor', Applied Materials Today, vol. 11, pp. 280-290.

See this record in the RMIT Research Repository at:

https://researchbank.rmit.edu.au/view/rmit:47651

Version: Accepted Manuscript

\section{Copyright Statement:}

(C) 2018. This manuscript version is made available under the CC-BY-NC-ND 4.0 license http://creativecommons.org/licenses/by-nc-nd/4.0/

\section{Link to Published Version:}

https://dx.doi.org/10.1016/j.apmt.2018.03.003 


\title{
Inducing tunable switching behavior in a single memristor
}

Taimur Ahmed, Sumeet Walia, Edwin L. H. Mayes, Rajesh Ramanathan, Paul Guagliardo, Vipul Bansal, Madhu Bhaskaran, J. Joshua Yang and Sharath Sriram

Mr. Taimur Ahmed, Dr. Sumeet Walia, Assoc. Prof. Madhu Bhaskaran, Assoc. Prof. Sharath Sriram

Functional Materials and Microsystems Research Group and Micro Nano Research Facility, RMIT University, Melbourne, VIC 3001, Australia

Dr. Edwin L. H. Mayes

RMIT Microscopy and Microanalysis Facility, RMIT University, Melbourne, VIC 3001, Australia

Dr. Paul Guagliardo

Centre for Microscopy, Characterisation and Analysis, The University of Western Australia, Perth, WA 6009, Australia

Dr. Rajesh Ramanathan, Prof. Vipul Bansal

Ian Potter NanoBioSensing Facility, NanoBiotechnology Research Laboratory, School of Science, RMIT University, Melbourne, VIC 3001, Australia

Prof. J. Joshua Yang Department of Electrical and Computer Engineering, University of Massachusetts, Amherst, MA 01003, USA

Correspondence emails:

taimur.ahmed@rmit.edu.au; sharath.sriram@rmit.edu.au

Keywords: resistive switching, valence change memory, doped $\mathrm{SrTiO}_{3}$, electroforming, current compliance, multiple switching

\begin{abstract}
Non-volatile resistive switching based on complex perovskite oxides have demonstrated an immense potential for future neuromorphic and compact logic applications. The adaptation of a functional oxide to exhibit different resistive switching characteristics is an important step in
\end{abstract}


harnessing the full suite of capabilities that such material system has to offer. Further, an understanding of the underlying phenomena that results in such adaptive characteristics is required. In this study, we show that multiple (threshold and bipolar) resistive switching behaviors can be achieved in a bilayer stack of titanium and selectively chromium-doped strontium titanate. High resolution transmission electron microscopic and electron energy loss

spectroscopic compositional and micro/nano-structural analyses reveal that the interfacial oxidation of the titanium layer to $\mathrm{Ti}_{2} \mathrm{O}_{3}$ introduces an oxide heterostructure with chromiumdoped strontium titanate. The concentration and distribution of oxygen vacancies in the heterostructure controls the switching behaviors, which can be controlled by defining the current compliance during the initial electroforming. The existence of current compliance dependent switching behaviors broadens the scope of applications of such selectively doped functional material for resistive memories by making them versatile thereby rendering the technology adaptable to different applications.

\section{Introduction}

Resistance change oxide memories have emerged as potential candidates for future nonvolatile memory applications owing to their distinct advantages over traditional charge-based memories. Their key features include excellent stability down to the sub-nm regime, high endurance, low power consumption and the ability to implement neuromorphic computing architectures [1-5]. To date, various resistive switching mechanisms have been proposed, which can be mainly classified as electrochemical metallization (ECM) and valance change mechanisms (VCM). The ECM represents the cation-based memory systems where electrochemical deposition and dissolution of metallic cations define the resistive switching whereas in VCM the resistive 
switching is attributed to the electric field induced redox reactions and migration of point defects (such as oxygen vacancies, $\mathrm{V}_{\mathrm{os}}$ ) [1]. Transition metal oxides are known to exhibit VCM based resistive switching and have demonstrated diverse switching behaviors - namely, unipolar, bipolar (BP) and complementary resistive switching (CRS) - which highlights the complex nature of VCM [6-12].

To date a variety of material systems, including metal-organic semiconductors [13, 14], binary metal oxides [5, 15-17] and transition metal oxides [18-21], have demonstrated resistive switching. Among the transition metal oxides, strontium titanate $\left(\mathrm{SrTiO}_{3}, \mathrm{STO}\right)-$ regarded as a model perovskite system - has shown enormous potential for VCM based resistive switching memory due to its versatile defect chemistry, mixed anionic-electronic and nonlinear switching kinetics $[1,10,22-24]$. The STO-based resistive memories are known to exhibit bipolar (BP) resistive switching behavior, but there are few reports on the transition between unipolar and $\mathrm{BP}$ resistive switching behaviors in a single memory cell $[25,26]$. Moreover, no clear and direct evidence is available that elaborates the switching behaviors and the driving mechanisms for this versatile functionality. Therefore, to achieve and precisely control the existence of more than one switching behaviors in the perovskite STO-based systems, insights into the structural and electrochemical factors are important to understand the multifunctional switching behaviors. Herein, we report the presence of BP (clockwise and counter-clockwise) and threshold (CRS and "peculiar" CRS) resistive switching behaviors in single units of chromium-doped amorphous oxygen-deficient STO (Cr: $a$-STO $x$ ) based metal-insulator-metal (MIM) devices. The distinct resistive switching behaviors are permanently induced by the current compliance limit and applied bias polarity during the initial electroforming step. The distinct presence of discrete switching behaviors in a simple two-terminal MIM device and their control by a single parameter 
(i.e., current compliance), offers an application based selection of the device behavior. The BP switching characteristics offer non-volatile data storage, whereas a threshold switching behavior offers selector-less bi-directional memory with multi-state data storage. Through detailed compositional and electrochemical analyses of the $\mathrm{Cr}: a$-STO $x$ MIM devices, the observed multiple resistive switching behaviors are attributed to the distribution and concentration of $V_{o s}$ generated, and their drift/diffusion under the influence of set current compliance. It is also observed that the locally-created crystalline regions (formed by the Joule heating) provide a localized conductive path for the anionic-electronic transport across the asymmetric switching MIM cell. Based on the electrical and material characterizations, a physical mechanism is also proposed to interpret the switching kinematics of the presented multiple resistive switching behaviors. Considering the advantages offered by the switching behaviors (BP and threshold resistive switching) demonstrated in our devices and their on-demand selectivity, this study is an important milestone in understanding and tuning multifunctional behaviors in a single STObased memory cell (and potentially transferrable to other oxides). This can significantly widen its applications in future high density memory and computational architectures

\section{Characterization of $\mathrm{Cr}: a-\mathrm{STO}_{x}$}

Fig. 1a illustrates a schematic of the $\mathrm{Cr}: a-\mathrm{STO}_{x}$ based MIM device fabricated in a cross-point structure where a stack of Ti $(8 \mathrm{~nm}) / \mathrm{Cr}: a-\mathrm{STO}_{x}(25 \mathrm{~nm})$ is sandwiched between Pt electrodes. In order to evaluate the chemical composition of sputtered Cr: $a$-STO $x$ thin films, comparative compositional analysis is carried out between Cr-doped and un-doped $a$-STO thin films by using high resolution X-ray photoelectron spectroscopy (XPS, see Experimental for details). The corelevel XPS spectra of Ti $2 p$ from Cr: $a$-STO $x$ (Fig. 1b) show two spin-orbit pairs (with splitting of 
$\sim 5.3 \mathrm{eV}$ ) and reveal the presence of two oxidation states i.e., $\mathrm{Ti}^{4+}$ and $\mathrm{Ti}^{3+}$ corresponding to the binding energies of $458.23 \mathrm{eV}$ and $456.22 \mathrm{eV}$ (both $2 p_{3 / 2}$ ), respectively [27, 28]. In addition, the core-level spectra of $\mathrm{O} 1 s$ and $\mathrm{Sr} 3 d$ show the presence of $\mathrm{O}^{2-}$ and $\mathrm{Sr}^{2+}$ oxidation states, respectively (Supporting Information, Fig. S1).

The resolved spectra of $\mathrm{Cr} 2 p_{3 / 2}$ (Fig. 1c) show two peaks at $576.72 \mathrm{eV}$ and $579.91 \mathrm{eV}$ which can be assigned to $\mathrm{Cr}^{3+}$ and $\mathrm{Cr}^{6+}$ oxidation states, respectively [29-31]. It has been reported that doping of $\mathrm{Cr}$ in STO can either incorporate the Cr ions at Ti or Sr sites [30, 31]. In our Cr:a$\mathrm{STO}_{x}$ thin films, a shift in the Ti $2 p$ peaks (Fig. 1b) towards lower binding energies (by $\sim 0.16 \mathrm{eV}$ ), as compared to the un-doped oxygen deficient $a-\mathrm{STO}_{x}$ (Supporting Information, Fig. S2), together with the presence of $\mathrm{Cr}^{6+}$ species suggest that the $\mathrm{Cr}$ doping mainly affects the Ti sites in $\mathrm{Cr}: a-\mathrm{STO}_{x}[30,31]$. Also, the $\mathrm{Cr}^{6+}$ prefers to accommodate at the Ti-site instead of Srsite. This is because the ionic radius of $\mathrm{Cr}^{6+}(0.0440 \mathrm{~nm})$ is similar to that of $\mathrm{Ti}^{4+}(0.0605 \mathrm{~nm})$. In contrast, the ionic radius of $\mathrm{Sr}^{2+}(0.1180 \mathrm{~nm})$ is much larger, which does not satisfy the limits of tolerance factor defined by Goldshmidt to retain the structure of an $\mathrm{ABO}_{3}$-type complex oxide [31]. Furthermore, the substitutional $\mathrm{Cr}$ doping in $a-\mathrm{STO}_{x}$ with a low concentration $(\mathrm{Cr} / \mathrm{Ti} \approx$ $0.025)$ is not expected to distort the chemical structure of $a$-STO $x$ perovskite oxide [32].

The electronic structure of $\mathrm{Cr}: a-\mathrm{STO}_{x}$ is assessed by obtaining the photoluminescence (PL) spectra at room temperature using a $325 \mathrm{~nm}(3.82 \mathrm{eV})$ laser excitation source. Fig. $1 \mathrm{~d}$ shows the PL spectra obtained from bare $\mathrm{Cr}: a-\mathrm{STO}_{x}$ thin films. A high intensity broad peak, centered at $535 \mathrm{~nm}(2.32 \mathrm{eV})$ was observed which could be de-convoluted into multiple emission bands. Such a broad emission at room temperature is characteristic of doped [33, 34] and un-doped $a$ STO thin films [35-37]. In un-doped $a$-STO thin films, room temperature PL emission is associated with the presence of $\mathrm{TiO}_{5}$ defects $[34,36,37]$. On the other hand, $\mathrm{Cr}$ is known to 
modify the electronic structure of STO by forming $\mathrm{CrO}_{5}$ defect structures with $\mathrm{V}_{\mathrm{o}} \mathrm{S}$ and introducing in-gap electronic states above the valance band edge [31, 34, 38-40]. The emission energy of $2.32 \mathrm{eV}$ in $\mathrm{Cr}: a-\mathrm{STO}_{x}$ (Fig. 1d) is comparatively lower than that of our previously reported un-doped $a-\mathrm{STO}_{x}$ (by $0.94 \mathrm{eV}$ ) at the same excitation energy [33] which indicates the presence of in-gap electronic states in $\mathrm{Cr}: a-\mathrm{STO}_{x}$. The XPS and PL analyses suggest that a low concentration $\mathrm{Cr}$ doping $(\mathrm{Cr} / \mathrm{Ti} \approx 0.025)$ in $a-\mathrm{STO}_{x}$, primarily influences the Ti sites, resulting in oxygen deficient $\left[\mathrm{TiO}_{5} \mathrm{~V}_{0}\right]$ and $\left[\mathrm{CrO}_{5} \mathrm{~V}_{0}\right]$ complexes that give rise to the in-gap electronic states [34]. Consequently, the introduction of these additional states can offer the possibility of manipulating the switching behaviors of our MIM devices using different applied electric fields, which is discussed later in the manuscript.

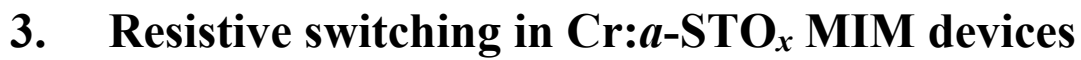

In order to activate the resistive switching in the STO based MIM devices, usually an initial high voltage sweep (higher than the subsequent switching threshold voltages) is required as an electroforming step which creates extended defects (i.e., nanofilament) through redox processes in the oxide $[1,10,27,41]$. In our Cr: $a-\mathrm{STO}_{x} \mathrm{MIM}$ devices, the multiple resistive switching characteristics have been achieved (regardless of the device area) by controlling the initial bias polarity and current compliance during the electroforming process (Supporting Information, Section S2). 


\subsection{Threshold switching}

\subsubsection{Complementary resistive switching}

The complementary resistive switching (CRS) is considered as one of the possible solutions to eliminate the sneak currents in a passive crossbar array for high density memory integration [42].

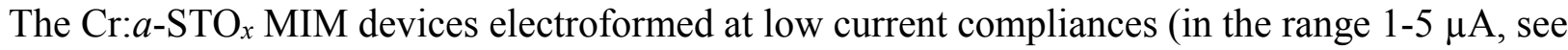
Supporting Information, Fig. S3) exhibit CRS behavior, as depicted in Fig. 2. A commonly known CRS cell comprises of two BP switching cells connected anti-serially through an intermediate electrode (in a tri-layer structure) and each cell can be reversibly switched between low resistive state (LRS) and high resistive state (HRS) under opposite applied voltage polarities. Unlike bipolar characteristics where two resistive states (HRS and LRS) define the device state, three states HRS-1, HRS- 0 , and ON represent the different configurations of the resistive states in the CRS operation [11, 42]. Both HRS-1 and HRS-0 states give high resistance at low READ voltages $\left(V_{R E A D}\right)$ than a certain threshold voltage (which is an intrinsic property of the CRS mechanism to suppress sneak currents in a memory array) while ON state gives low device resistance at high $V_{R E A D}$.

In our $\mathrm{Cr}: a-\mathrm{STO}_{x} \mathrm{MIM}$ devices, transition of resistive states from HRS- 1 to ON and from ON to HRS-0 occurs at positive threshold voltages during cyclic voltage sweeps and are denoted by $V_{T H, 1}$ and $V_{T H, 2}$, respectively, as shown in Fig 2a. Similarly, the transition from HRS-0 to ON and from ON to HRS-1 occurs at negative threshold voltages as denoted by $V_{T H, 3}$ and $V_{T H, 4}$ respectively. In CRS, both HRS- 1 and HRS-0 are regarded as HRS at low $V_{R E A D}$ (between $V_{T H, 3}$ and $V_{T H, I}$ ) whereas ON is a transition state (between HRS-1 and HRS-0) that separates resistive states at higher $V_{R E A D}$ (such as $V_{T H, 1}<V_{R E A D}<V_{T H, 2}$ or $V_{T H, 3}<V_{R E A D}<V_{T H, 4}$ ) [11, 42]. Fig. 2b 
shows the statistical distribution of $V_{T H, 1}$ and $V_{T H, 3}$ for 1000 cycles and the average values are calculated to be $1.43 \mathrm{~V}$ and $-1.65 \mathrm{~V}$, respectively.

\subsubsection{Peculiar complementary resistive switching}

In a conventional readout operation of CRS devices, generally a higher $V_{R E A D}$ (than transitional thresholds $V_{T H, l}$ and $V_{T H, 3}$ ) is required, as the stored states (HRS-1 and HRS-0) cannot be distinguished at lower $V_{R E A D}$. This can change the resistive state of the CRS device and rewriting of the resistive state is required as a result of the destructive readout operation [43, 44]. Interestingly, our Cr: $a$-STO $x$ MIM devices, electroformed at moderate current compliance limits (in range 10-15 $\mu \mathrm{A}$, see Supporting Information Fig. S3), show a reproducible "peculiar" CRS ( $p$-CRS) behavior Fig. 3a, where the stored resistive states can be distinguished at lower read voltage (i.e., $V_{R E A D, l}$ ) in a non-destructive readout. The prominent feature of $p$-CRS (as compared to the conventional CRS) is the possibility of three-level memory states where LRS, HRS and ON can be distinctively stored in a single cell and the inherent switchable diode characteristics of $p$-CRS can facilitate the implementation of complete logic operations [45]. As depicted in Fig. 3a, the devices switch the states from LRS to ON at $V_{T H, I}$ and from ON to HRS at $V_{T H, 2 .}$ Also the transition from HRS to ON occurs at $V_{T H, 3}$ and $\mathrm{ON}$ to LRS at $V_{T H, 4}$. The average values of transition thresholds $V_{T H, 1}$ and $V_{T H, 3}$ are calculated to be $+1.45 \mathrm{~V}$ and $-1.35 \mathrm{~V}$, respectively, from their statistical distribution (Fig. 3b) and the values of $V_{T H, 2}$ and $V_{T H, 4}$ are $+1.8 \mathrm{~V}$ and $1.8 \mathrm{~V}$, respectively.

Fig. $3 \mathrm{c}$ shows the retention of the $p$-CRS regime for $10^{5} \mathrm{~s}$. Constant READ voltages $V_{R E A D, 1}$ of $+0.2 \mathrm{~V}$ and $V_{R E A D, 2}$ of $+1.6 \mathrm{~V}$ are used to measure the HRS/LRS and ON state resistances, respectively. Distinguishable HRS and LRS with an average switching ratio (HRS/LRS) of $\sim 9$ 
suggests the capability and stability of bi-level storage of the $p$-CRS behavior in our Cr: $a$-STO $x$ MIM devices. Fig. $3 \mathrm{~d}$ shows the endurance characteristics of the $p$-CRS regime for $<5000$ switching cycles. The loss of resistive states after $4.3 \times 10^{3}$ cycles can be attributed to the cycling-induced degradation due to relatively higher $V_{R E A D, 2}$ and dual $V_{S E T}$ pulses in each cycle (as depicted inset of Fig. 3d) [46, 47]. During the endurance measurements, a train of short pulses with a duration of $50 \mu$ s are used to WRITE/ERASE/READ the devices (inset of Fig. 3d) For each complete WRITE/ERASE/READ cycle, a positive pulse with an amplitude of $+2.3 \mathrm{~V}$ switches the devices to HRS followed by a $V_{R E A D, l}$ pulse with an amplitude of $+0.2 \mathrm{~V}$. A negative pulse of $-2.3 \mathrm{~V}$ amplitude switches the devices to LRS/ON followed by $V_{R E A D, I}(+0.2 \mathrm{~V})$ and $V_{R E A D, 2}(+1.6 \mathrm{~V})$ pulses to READ the LRS and ON states, respectively. Finally, a second negative pulse of $-2.3 \mathrm{~V}$ is required to restore the LRS/ON states, prior to the next cycle. The $p$ CRS characteristics are evaluated for more than 15 devices which reveal that our Cr: $a$-STO $x$ MIM devices exhibit reproducible $p$-CRS behavior (Section S2, Supporting Information, Fig. S5).

\subsection{Bipolar resistive switching}

As depicted in Fig. 4,, two different electroforming-polarity dependent BP resistive switching behaviors (see Supporting Information, Fig. S3) are observed in our Cr: $a$-STO $x$ MIM devices. Both BP resistive switching behaviors are induced at higher current compliances than the CRS and $p$-CRS during the initial electroforming step. While applying positive bias on the top electrode, the electroforming processes with current compliance in 40-100 $\mu \mathrm{A}$ range, sets the Cr: $a$-STO $x$ MIM devices into the clockwise $\mathrm{BP}(\mathrm{CW}-\mathrm{BP})$ resistive switching regime. Fig. $4 \mathrm{a}$ shows repeatable and well-defined CW-BP switching cycles which are a signature of $a$-STO devices (RESET on positive bias and SET on negative bias) [10,27]. Fig. 4b shows stable 
retention characteristics for $10^{5} \mathrm{~s}$ with an average switching ratio (HRS/LRS) of $\sim 19$ at a constant $V_{R E A D}$ of +0.2 V. Fig. $4 \mathrm{c}$ shows the endurance of MIM devices (pre-set into CW-BP switching behavior) for $10^{4}$ cycles. On the other hand, applying positive bias on the bottom electrode during the electroforming process (with current compliance in 40-100 $\mu \mathrm{A}$ range) induces a counter-clockwise BP (CCW-BP) resistive switching (Fig. 4d). The retention of CCWBP switching behavior is measured for $10^{5} \mathrm{~s}$ (Fig. 4e) and shows no degradation with an average switching ratio of $\sim 25$ at a constant $V_{R E A D}$ of +0.2 V. Fig. $4 \mathrm{f}$ shows endurance of CCW-BP switching behavior for $10^{4}$ cycles.

The existence of multiple resistive switching behaviors (in identical MIM devices) suggests that the intrinsic Schottky contact at the bottom Cr:a-STO $/$ Pt interface $[48,49]$ and an expected Ohmic contact at the top Pt/Ti/Cr: $a-\mathrm{STO}_{x}$ interface [50] are modified under the influence of different current compliance limits and applied polarities during the electroforming step [19]. The oxidation of the top Ti layer into a sub-stoichiometric titanium oxide (as discussed in the following sections) introduces another functional switching layer at the top interface which contributes in defining and executing the multiple resistive switching behaviors. In order to understand the role of the top Ti layer, symmetric Pt/Cr: $a-\mathrm{STO}_{x} / \mathrm{Pt} \mathrm{MIM}$ devices were fabricated (Section S2, Supporting Information, Fig. S6). Regardless of the current compliance and applied polarity during the initial electroforming, no significant and well defined resistive switching characteristics are observed. This further indicates that the presence of Ti at the top interface renders our devices with the asymmetry which is responsible for the observed multiple resistive switching behaviors. 


\section{Spectroscopic analyses of the Cr:a-STO $x$ MIM devices}

\subsection{Secondary ion mass spectroscopy (SIMS)}

In the electroforming process, isolated incomplete filaments can simultaneously be formed over the electroforming cathode along with a fully extended nanofilament in a MIM device (as shown in Fig. 3 of Ref.[51]). A depth profile analysis can indicate the switching mechanism through the distribution of principle elements, without distorting their oxidation states, throughout the thickness of MIM device. The dynamic secondary ion mass spectroscopy (SIMS) depth profiles are used to assess the distribution of principal elements across the Cr: $a$-STO $x$ MIM devices. The raw depth profile data, shown in Fig. 5, depicts the distribution of $\mathrm{O}^{-}, \mathrm{TiO}^{-}, \mathrm{SrO}^{-}$and $\mathrm{CrO}^{-}$ species across three different MIM devices in three different states, namely - pristine, LRS and HRS, as a function of sputtering time during analyses. Also, the switching MIM devices were pre-set to exhibit CW-BP resistive switching behavior and subjected to $I-V$ switching cycles (at least 100 cycles). An increase in signals for the $\mathrm{O}^{-}$and $\mathrm{TiO}^{-}$species at the top $\mathrm{Ti} / \mathrm{Cr}: a-\mathrm{STO}_{x}$ interface of the pristine MIM device indicates the partial oxidation of the Ti layer. This is consistent with our cross-sectional compositional analyses (discussed later in the manuscript) and earlier XPS depth profile analyses of the switching MIM devices [27]. The $\mathrm{O}^{-}$profile in the pristine and LRS shows a uniform distribution of oxygen across the Cr: $a$-STO $x$ layer. However, for the device set in the HRS, the $\mathrm{O}^{-}$profile clearly shows that the oxygen content drops at the bottom $\mathrm{Cr}: a-\mathrm{STO}_{x} / \mathrm{Pt}$ interface. This indicates that in HRS relatively more $\mathrm{V}_{\mathrm{oS}}$ accumulate at the vicinity of the bottom electrode than the top interface. The relative differences in LRS and HRS profiles suggest that the drift of $\mathrm{V}_{\mathrm{o}}$ s under the reverse bias polarities and nano-redox reactions induce the change in resistive states $[1,15,52,53]$. 


\subsection{Cross-sectional structural and compositional analyses}

In order to establish the resistive switching mechanism in the Cr: $a-\mathrm{STO}_{x} \mathrm{MIM}$ devices, high resolution transmission electron microscope (HR-TEM) and electron energy loss spectroscopic (EELS) analyses are carried out. As expected, the TEM micrographs of the pristine MIM device show an amorphous microstructure of the functional oxide layer along the length of the device cross-sections, owing to the room temperature sputtering of $\mathrm{Cr}: a-\mathrm{STO}_{x}$ thin film (as shown in Fig. 1 of Ref. [51]). Also, the cross-sectional EELS analysis of the pristine MIM device reveals the partial oxidation of the top Ti layer and presence of a sub-stoichiometric titanium oxide layer at the top Ti/Cr: $a-\mathrm{STO}_{x}$ interface (as depicted in Fig. 2 of Ref. [51]).

The HR-TEM micrographs of the MIM devices exhibiting BP resistive switching characteristics illustrate the presence of localized crystalline regions in the functional $\mathrm{Cr}: a-\mathrm{STO}_{x}$ layer. To analyze the structure and composition of these localized crystalline regions and the metal/oxide interfaces, regions of interest (ROIs) were identified in the lamellae prepared from MIM devices pre-set into LRS. In the MIM devices exhibiting CCW-BP resistive switching behavior, a distinguishable high contrast ROI in the bulk amorphous functional oxide layer (highlighted in Fig. 6a) is selected, which contains a crystalline region emanating from the bottom Pt electrode. Similar localized crystalline regions are also observed in MIM devices pre-set to HRS of CW-BP behavior prior to TEM sample preparation (as shown in Fig. 5 of Ref. [51]). The fast Fourier transform (FFT) diffraction pattern (Fig. 6b) of the ROI shown in Fig. 6a is generated to determine the constituent phases of the crystalline structures. The high intensity diffraction spots marked as spot 1 and spot 2 in Fig. 6b can be assigned to the cubic STO phase. Spot 1 with the $d$ spacing of $1.9 \AA$ is used to generate the inverse FFT (Fig. 6c) of the ROI which outlines the predominant existence of the [200] cubic STO phase in the Cr: $a$-STO $x$ layer. Spot 2 with the $d$ - 
spacing of $2.1 \AA$ matches the inter-planar spacing of [111] cubic STO and has a relatively less volume ratio (as observed from the extracted inverse FFT, not shown for brevity). It should be noted that due to our instrumental limitations and experimental conditions we cannot exclude the presence of other sub-stoichiometric STO phases in the ROI [54, 55] (see Fig. 5 of Ref. [51]).

Now, we assess the top interface. The ROI shown in Fig. $6 \mathrm{~d}$ depicts a crystalline region at the top Ti/Cr: $a-\mathrm{STO}_{x}$ interface of the switching MIM device pre-set in a LRS prior to sample preparation. The respective FFT diffraction patterns (Fig. 6e) are used to index the existing phase in the ROI. The highest intensity diffraction spot marked as spot 3, with the $d$-spacing of $2.1 \AA$, is used to outline the crystalline region in the inverse FFT micrograph (Fig. 6f) and could be indexed to a [200] rhombohedral $\mathrm{Ti}_{2} \mathrm{O}_{3}$ phase. This suggests that the top Ti layer changes its microstructure from as-deposited amorphous to crystalline after the MIM devices are subjected to the electroforming (see Fig. 4 of Ref. [51]) and subsequent switching cycles [56, 57]. Moreover, the electric field induced oxygen evolution in the $\mathrm{Cr}: a-\mathrm{STO}_{x}$ layer and its diffusion into the Ti layer during continues cyclic switching and electrical stress results in a gradual increase in the thickness of the sub-stoichiometric phase (such as $\mathrm{Ti}_{2} \mathrm{O}_{3}$ ) at the top Ti/Cr: $a-\mathrm{STO}_{x}$ interface $[33,58,59]$. The formation of $\mathrm{Ti}_{2} \mathrm{O}_{3} / \mathrm{Cr}: a-\mathrm{STO}_{x}$ oxide heterostructure explains the observed threshold resistive switching behavior in our MIM devices, as discussed in the next section.

To assess the relative differences in the electronic structure of the bulk amorphous and the locally crystalline ROIs, EELS spectra from cross-sectional line and area scans were acquired from switching devices. The fine structures of $\mathrm{Ti}-L_{2,3}$ and $\mathrm{O}-K$ edges are used to analyze the oxidation states of titanium and the distribution of oxygen content, respectively, across the MIM devices. Considering the instrument limitations, we restrict to a qualitative analysis only instead 
of quantifying the relative changes in the electronic structure. Fig. 7a shows the EELS area map of the locally crystalline ROI (indicated in Fig. 6a) to highlight the relative distribution of oxygen content across the MIM device after the cyclic CCW-BP switching. The EELS area map is generated by considering the $\mathrm{O}-K$ edge intensities of the collected spectra (at each pixel) after pre-edge background subtraction. It reaffirms the observations of the FFT analysis which showed that the Ti layer at the top Ti/Cr: $a$-STO $x$ interface (Fig. 6e) oxidizes to sub-stoichiometric $\mathrm{Ti}_{2} \mathrm{O}_{3}$ phase due to the electrochemical redox reactions. The relatively lower oxygen concentration in the upper region of the ROI indicates the sub-stoichiometric nature (oxygen-deficient) of the top Ti/Cr: $a-\mathrm{STO}_{x}$ interface relative to the bottom $\mathrm{Cr}: a-\mathrm{STO}_{x} / \mathrm{Pt}$ interface. Furthermore, the oxygen deficient regions (i.e., $\mathrm{V}_{\mathrm{o}} \mathrm{s}$ ) extending from top Pt electrode (acting as cathode), through the Cr: $a$ $\mathrm{STO}_{x}$ layer towards the bottom Pt electrode (acting as anode), indicate the filamentary path across the MIM structure in the LRS. Spatially isolated oxygen deficient hotspots in the bulk Cr: $a-\mathrm{STO}_{x}$ layer, identified in the EELS map, indicate the oxygen deficient stoichiometry of the functional oxide which is explained earlier in the XPS analysis (Fig. 1). The presence of oxygen content within the top and bottom Pt electrodes can be attributed to the out-diffusion of oxygen ions along the Pt grain boundaries during the cyclic switching [60, 61]. In order to assess the distribution of $\mathrm{V}_{\mathrm{oS}}$ in the HRS of CW-BP behavior, the EELS area map of the ROI (indicated in Fig. 5 of Ref. [51]) shows the accumulation of $V_{o}$ at the anode without their extension to the cathode (see Fig. 6 of Ref. [51]). Based on the observed distribution of $V_{o s}$ in LRS and HRS, it can be inferred that the bias polarity induced migration of $\mathrm{V}_{\mathrm{oS}}$ and the associated formation and rupture of the oxygen deficient path is responsible for the switching in our Cr:a-STO $x$ MIM devices. In the following section, we provide further evidence that indicates that these oxygen 
deficient pathways are the conductive routes for the charge transportation due to the change of $\mathrm{Ti}$ valance from $\mathrm{Ti}^{4+}$ to $\mathrm{Ti}^{3+}$.

Fig. $7 \mathrm{~b}$ depicts the relative change in the fine structures of the background-subtracted $\mathrm{Ti}-L_{2,3}$ and $\mathrm{O}-K$ edges along the EELS cross-sectional line scans passing over the locally crystalline ROI highlighted in Fig. 6a. The edge profiles at the distinct points such as the top Pt/Ti interface, Ti/Cr: $a-\mathrm{STO}_{x}$ interface, center of the oxide layer and at the bottom $\mathrm{Cr}: a-\mathrm{STO}_{x} / \mathrm{Pt}$ interface are highlighted. The gradual evolution of the Ti oxidation states from the top Pt/Ti interface to the bottom Pt electrode are clearly observed in the Ti- $L_{2,3}$ edge profiles. On the other hand, $\mathrm{O}-K$ and $\mathrm{Cr}-L_{2,3}$ edge spectra collected from the EELS line scan are weak and exhibit low signal-to-noise ratio which can be associated with the resolution limit of the instrument and low doping concentration of $\mathrm{Cr}(\mathrm{Cr} / \mathrm{Ti} \approx 0.025)$. So we rely on the $\mathrm{Ti}-L_{2,3}$ edge profiles to qualitatively analyze the electronic structure of the locally crystalline ROI. Since each $\mathrm{V}_{\mathrm{o}}$ introduces two electrons into the Ti $3 d$ orbital, the resulting change in the Ti valence band can be observed from the Ti- $L_{2,3}$ edge $[53,62]$. At the top Pt/Ti interface, the Ti- $L_{2,3}$ profiles with broad peaks and relatively weak intensities indicate the presence of mixed $\mathrm{Ti}^{2+}$ and $\mathrm{Ti}^{3+}$ oxidation states $[58,63$, 64]. At the Ti/Cr: $a$-STO $x$ interfacial region, the crystal-field splitting of the Ti- $L_{3}$ and Ti- $L_{2}$ peaks into $t_{2 \mathrm{~g}}$ and $e_{\mathrm{g}}$ peaks which can be attributed to the presence of $\mathrm{Ti}^{3+}$ and $\mathrm{Ti}^{4+}$ oxidation states $[58,63,65]$. However, as we move away from the top interface and towards the bottom, more pronounced $t_{2 \mathrm{~g}}$ and $e_{\mathrm{g}}$ peaks indicate the dominant presence of the $\mathrm{Ti}^{4+}$ oxidation state. The change in Ti oxidation states across the MIM device suggest that the top Pt/Ti/Cr: $a-\mathrm{STO} x$ interface is more oxygen deficient compared to the bottom $\mathrm{Cr}: a-\mathrm{STO}_{x} / \mathrm{Pt}$ interface which is consistent with the EELS O- $K$ area map of the ROI (Fig. 7a). 


\section{Mechanism of resistive switching in Cr:a-STO $x$ MIM devices}

Based on the extensive material and interfacial characterization as well as the electrical characteristics, we propose a mechanism for the unique behavior of our Cr:a-STO $x$ MIM devices. The inherent $\mathrm{V}_{0}$ in the $\mathrm{Ti}-\mathrm{O}_{6}$ octrahedra that are created by preferentially removing the oxygen atoms due to the heavy $\mathrm{Ar}^{+}$ion bombardment during the sputtering process [27], generate conduction band electrons in the Ti $3 d$ states. However, doping with $\mathrm{Cr}$ (via co-sputtering from a metallic $\mathrm{Cr}$ target) localizes the conduction band electrons by trapping them at $\mathrm{Cr}$ sites, which reduces the $\mathrm{Cr}$ from $\mathrm{Cr}^{4+}$ to $\mathrm{Cr}^{3+}$ species $[66,67]$. Consequently, no conduction electrons are introduced in the Ti $3 d$ states and therefore the pristine $\mathrm{Cr}: a$-STO $x$ oxide exhibits an insulating nature. Prior to the electroforming, all $\mathrm{Cr}: a-\mathrm{STO} x$ MIM devices exhibit device resistances of $>1 \mathrm{G} \Omega$. It is widely accepted that the electroforming process generates $V_{o s}$ and alters their distribution in metal oxides through subsequent electrochemical redox processes $[1,2,16,52]$. Consequently, a localized channel for current flow through the oxide system is created which is essentially an oxygen deficient filamentary pathway. Moreover, the subsequent resistive switching is attributed to the rapture and formation of the filamentary path at its weakest part, most likely close to the electroforming anode [7], through the Joule heating and drift of $V_{o s}$ under the influence of applied bias.

In our Cr: $a$-STO $\mathrm{SIM}_{x}$ devices, $\mathrm{Cr}$ doping further increases the concentration of $\mathrm{V}_{\mathrm{oS}}$ which facilitates the formation of a conductive filamentary path during the electroforming process [68]. The locally confined current flow through the filamentary pathway may induce local Joule heating causing the formation of locally extending crystalline regions in the bulk amorphous Cr: $a-\mathrm{STO}_{x}$ layer. Also, the top Ti layer (that develops into a sub-stoichiometric $\mathrm{Ti}_{2} \mathrm{O}_{3}$ layer) introduces an additional switching layer that is electrochemically different to the $\mathrm{Cr}: a-\mathrm{STO}_{x}$. 
This causes a change in the mobility [69] and formation energies of $\mathrm{V}_{\mathrm{oS}}$ [70-72]. $\mathrm{Ti}_{2} \mathrm{O}_{3}$ is known to exhibit semiconductor-metal transition at an elevated temperature and is potentially responsible for the threshold resistive switching in our MIM devices. During the switching cycles, the Joule heating produced by the electrical bias switches the $\mathrm{Ti}_{2} \mathrm{O}_{3}$ to its conducting phase resulting in a minimal electric field drop on $\mathrm{Ti}_{2} \mathrm{O}_{3}$ and as such it sustains the electrical bias without further reduction. Therefore, the current compliance and bias polarity dependent generation of $\mathrm{V}_{\mathrm{oS}}$ and the formation of filamentary paths in the $\mathrm{Ti}_{2} \mathrm{O}_{3} / \mathrm{Cr}: a-\mathrm{STO}_{x}$ oxide heterostructure govern the multiple resistive switching behaviors in our $\mathrm{Cr}: a$-STO $x$ MIM devices. When the electroforming bias is applied across the MIM devices the applied electric field initializes the generation of $\mathrm{V}_{\mathrm{o}}$ which is driven by the movement of oxygen ions towards the anode and several localized spots, including Cr doped sites, becoming oxygen deficient [73]. Under the influence of applied electroforming bias, the positively charged $V_{o s}$ drift towards the electroforming cathode and start to accumulate at its vicinity whereas their density extends towards the anode, forming an extended oxygen deficient filamentary pathway.

At lower current compliances (i.e., 1-15 $\mu \mathrm{A}$ ) during the electroforming, relatively less density of $V_{o s}$ are generated [73]. Also the insufficient driving force for their migration prohibits the complete formation of the filamentary pathway across the $\mathrm{Ti}_{2} \mathrm{O}_{3} / \mathrm{Cr}: a-\mathrm{STO}_{x}$ interface. This results in the formation of two distinctive filamentary paths in the $\mathrm{Ti}_{2} \mathrm{O}_{3}$ and $\mathrm{Cr}: a-\mathrm{STO}_{x}$ layers. Depending on the bias polarity, each layer switches (to LRS and HRS) individually to define the resistive state of the MIM device. Under these conditions, we explain the threshold switching behavior in our MIM devices by the repetition of processes schematically depicted in the bottom panel of Fig. 8. Post-electroforming electrical characteristics of the CRS and $p$-CRS behaviors 
suggest that both resistive switching behaviors follow a similar switching mechanism in terms of $\mathrm{V}_{\mathrm{o}}$ distribution and migration in $\mathrm{Ti}_{2} \mathrm{O}_{3}$ and $\mathrm{Cr}: a-\mathrm{STO}_{x}$ layers.

On the other hand, electroforming at higher current compliances (40-100 $\mu \mathrm{A})$ generate a higher density of $V_{o}$ and sufficient driving force is available for their migration across the $\mathrm{Ti}_{2} \mathrm{O}_{3} / \mathrm{Cr}: a-\mathrm{STO}_{x}$ interface. The top panel in Fig. 8 schematically depicts the subsequent mechanism of the CCW-BP resistive switching behavior where the complete formation of extended oxygen-deficient filamentary path across the $\mathrm{Ti}_{2} \mathrm{O}_{3} / \mathrm{Cr}: a$-STO $\mathrm{ST}_{x}$ corresponds to the LRS and ruptured filamentary path (under reverse bias) set the HRS in MIM devices. Similar switching mechanism with opposite bias polarities can be applied to explain the CW-BP resistive switching behavior.

\section{Conclusion}

In conclusion, we report the presence of bipolar and threshold resistive switching behaviors in a single-layered $\mathrm{Pt} / \mathrm{Ti} / \mathrm{Cr}: a-\mathrm{STO}_{x} / \mathrm{Pt}$ memory device. The multiple switching behaviors are defined by the current compliance limit during the initial electroforming step. The electroforming polarity-dependent BP and threshold resistive switching behaviors are also presented. In order to understand the existence of multiple resistive switching behaviors, rigorous compositional and micro/nano-structural analyses were carried out on the bare $\mathrm{Cr}: a$-STO $x$ oxide and cross-sectional MIM devices. It was observed that top Ti layer oxidized to a sub-stoichiometric oxide under the applied bias and introduced a $\mathrm{Ti}_{2} \mathrm{O}_{3} / \mathrm{Cr}$ : $a-\mathrm{STO} x$ heterostructure in the MIM devices. Joule heating created local crystalline regions, providing a route for the migration of $V_{o s}$ across the oxide heterostructure. Finally, to explain the observed multiple switching behaviors, a physical mechanism is proposed where current compliance during electroforming defines the concentration and distribution of $\mathrm{V}_{\mathrm{os}}$ in the heterostructure and the subsequent switching 
behaviors are combined effect of local redox reactions and Joule heating. The existence of multiple resistive switching behaviors in a $\mathrm{Cr}: a-\mathrm{STO}_{x}$ based memory cell and their addressability through a single physical parameter, i.e., the current compliance during electroforming, opens new pathways for incorporation of memristors in ultra-dense memory architectures and conventional/non-conventional computation.

\section{Experimental}

Material deposition and device fabrication: The $a-\mathrm{Cr}: \mathrm{STO}_{x}$ resistive switching devices in cross-point configuration are fabricated via standard photolithography/lift-off and thin film deposition processes. After patterning through a photolithography (chrome) mask, the bottom Pt $(7 \mathrm{~nm}) / \mathrm{Ti}(3 \mathrm{~nm})$ electrodes are evaporated onto a $\mathrm{SiO}_{2}(300 \mathrm{~nm}) / \mathrm{Si}$ substrate by electron beam evaporation (Kurt J. Lesker PVD75 Pro-line). A $25 \mathrm{~nm}$ thin film of amorphous $\mathrm{Cr}$ doped oxygen-deficient STO is sputtered (Kurt J. Lesker PVD75 sputtering system) in a pure argon atmosphere at room temperature from a commercial ceramic STO target $(99.95 \%$, Testbourne Ltd) by using $200 \mathrm{~W}$ RF (13.54 MHz) plasma and under a sputtering pressure of $3.5 \times 10^{-3}$ Torr. Cr was incorporated into STO by co-sputtering of metallic Cr target (99.95\%, Testbourne Ltd) by using $4 \mathrm{~W}$ DC power. Detailed description on sputtering $a$-STO oxides with different stoichiometries conditions can be found in our earlier report [24, 27]. Finally, following the photolithographic patterning, top $\mathrm{Pt}(35 \mathrm{~nm}) / \mathrm{Ti}(8 \mathrm{~nm})$ electrodes are evaporated by electron beam evaporation at $<5 \times 10^{-7}$ Torr base pressure. The $a$-Cr:STO $x$ MIM cross-point devices with active area of $2 \times 2 \mu \mathrm{m}^{2}, 4 \times 4 \mu \mathrm{m}^{2} 10 \times 10 \mu \mathrm{m}^{2}, 20 \times 20 \mu \mathrm{m}^{2}, 40 \times 40 \mu \mathrm{m}^{2}, 80 \times 80 \mu \mathrm{m}^{2}$ and $100 \times 100 \mu \mathrm{m}^{2}$ are fabricated. 
Electrical characterization: The electrical characterizations of $\mathrm{Cr}: a-\mathrm{STO}_{x} \mathrm{MIM}$ devices are performed using an Agilent 2912A sourcemeter for two-probe measurements.

$X$-ray photoelectron spectroscopy: X-ray photoelectron spectroscopy (XPS) analysis is conducted by using a Thermo Scientific K-Alpha instrument utilizing an aluminum $K \alpha$ radiation source $(1486.6 \mathrm{eV})$ is used. The XPS spectra are collected from bare o (Cr-doped and un-doped) $a$-STO oxide thin films, sputtered on $\mathrm{SiO}_{2} / \mathrm{Si}$ substrates, after in-situ argon ion assisted surface cleaning step. Being aware of the fact that the argon ions can reduce the Ti oxidation states, we initially characterized the argon ion damage to minimize the preferential oxygen removal. By using a stoichiometric crystalline STO substrate it was found that a short $(<5 \mathrm{~s})$ argon milling step with low beam energy $(<200 \mathrm{eV})$ caused minimal oxygen removal. All spectra were resolved by using the standard Gaussian-Lorentzian function followed by the Shirley background correction.

Photoluminescence spectroscopy: The photoluminescence emission spectra are obtained using a Horiba Scientific FluoroMax-4 spectrofluorometer. All spectra are collected at room temperature from as-deposited bare $\mathrm{Cr}: a-\mathrm{STO}_{x}$ thin films sputtered on $\mathrm{SiO}_{2} / \mathrm{Si}$ substrates. A laser source with $325 \mathrm{~nm}$ of wavelength is used to excite the sputtered oxides.

Secondary ion mass spectroscopy: Dynamic secondary ion mass spectrometry (SIMS) analyses are carried out with a CAMECA NanoSIMS 50L. Elemental depth profiles are obtained using a $\mathrm{Cs}^{+}$primary ion source with a beam diameter of approximately $100 \mathrm{~nm}$, impact energy of $16 \mathrm{keV}$ and a beam current of approximately $3 \mathrm{pA}$. The instrument is operated in multi-collector mode, allowing the simultaneous detection of $\mathrm{O}^{-}, \mathrm{TiO}^{-}, \mathrm{SrO}^{-}$and $\mathrm{CrO}^{-}$. The raster size is $15 \mu \mathrm{m}^{2}$, however, only secondary ions from the central $10 \mu \mathrm{m}^{2}$ are used to determine the depth profiles. 
The mass spectrometer is calibrated using a commercial stoichiometric $\mathrm{SrTiO}_{3}$ single-crystal (100) substrate and pure Ti and Cr metals, prior to the characterization of the MIM devices.

Cross-sectional analyses: The transmission electron microscopy (TEM) and electron energy loss spectroscopic (EELS) analyses are performed on pristine, electroformed and switching (at least for 100 cycles and subjected to constant bias stresses) $\mathrm{Cr}$ : $a$-STO $x$ MIM devices using a JEOL 2100F scanning transmission electron microscope (STEM) with attached Tridium Gatan image filter with an entrance aperture of $5 \mathrm{~mm}$. Thin TEM lamellae are prepared by focused ion beam (FIB) cuts through the MIM structure by using a FEI Scios DualBeam ${ }^{\mathrm{TM}}$ system. Cross-sectional STEM micrographs and EELS spectra are collected using a $<1.5 \mathrm{~nm}$ beam spot. EELS spectra are collected with a dispersion of $0.3 \mathrm{eV}$ per pixel which allowed simultaneous recording of the titanium $L_{2,3}\left(\mathrm{Ti}-L_{2,3}\right)$ edge and oxygen $K(\mathrm{O}-\mathrm{K})$ edge in the regions of interest (ROIs) and across the MIM cross-sections. A power law fit is adopted for the pre-edge background correction while the influence of nearby peaks and plural scattering are reduced by narrow signal windows.

\section{Acknowledgements}

The authors acknowledge support from the Australian Research Council (ARC) for personnel and project support via DP130100062 (S.S.), DE160100023 (M.B.), and FT140101285 (V.B.) and equipment funding through LE0882246, LE0989615, LE110100223, and LE150100001. The authors would like to acknowledge the technical assistance of the Micro Nano Research Facility (MNRF) and the RMIT Microscopy and Microanalysis Research Facility (RMMF). Also Australian Microscopy \& Microanalysis Research Facility, AuScope, the Science and Industry Endowment Fund, and the State Government of Western Australian for contributing to the Ion Probe Facility at the Centre for Microscopy, Characterisation and Analysis at the University of Western Australia. 


\section{References}

1. Waser, R., et al., Redox-Based Resistive Switching Memories - Nanoionic Mechanisms, Prospects, and Challenges. Adv. Mater., 2009. 21(25-26): p. 2632-2663.

2. Waser, R. and M. Aono, Nanoionics-based resistive switching memories. Nat. Mater., 2007. 6(11): p. 833-840.

3. Wang, Z., et al., Memristors with diffusive dynamics as synaptic emulators for neuromorphic computing. Nat. Mater., 2017. 16(1): p. 101-108.

4. Yang, J.J., D.B. Strukov, and D.R. Stewart, Memristive devices for computing. Nat. Nanotechnol., 2013. 8(1): p. 13-24.

5. Lee, M.J., et al., A fast, high-endurance and scalable non-volatile memory device made from asymmetric $\mathrm{Ta}_{2} \mathrm{O}_{5-x} / \mathrm{TaO}_{2-x}$ bilayer structures. Nat. Mater., 2011. 10(8): p. 625-30.

6. Chen, J.Y., et al., Switching Kinetic of VCM-Based Memristor: Evolution and Positioning of Nanofilament. Adv. Mater., 2015. 27(34): p. 5028-33.

7. Yang, J.J., et al., Metal oxide memories based on thermochemical and valence change mechanisms. MRS Bulletin, 2012. 37(02): p. 131-137.

8. Kwon, D.H., et al., Atomic structure of conducting nanofilaments in $\mathrm{TiO}_{2}$ resistive switching memory. Nat. Nanotechnol., 2010. 5(2): p. 148-53.

9. Yoon, K.J., et al., Evolution of the shape of the conducting channel in complementary resistive switching transition metal oxides. Nanoscale, 2014. 6(4): p. 2161-9.

10. Nili, H., et al., Nanoscale Resistive Switching in Amorphous Perovskite Oxide (a-SrTiO3) Memristors. Adv. Func. Mater., 2014. 24(43): p. 6741-6750.

11. Breuer, T., et al., Low-current operations in $4 F^{2}$-compatible Ta ${ }_{2} \mathrm{O}_{5}$-based complementary resistive switches. Nanotechnology, 2015. 26(41): p. 415202.

12. Balatti, S., et al., Multiple memory states in resistive switching devices through controlled size and orientation of the conductive filament. Adv. Mater., 2013. 25(10): p. 1474-8.

13. Ramanathan, R., et al., Solution-processable do-it-yourself switching devices (DIY devices) based on CuTCNQ metal-organic semiconductors. Appl. Mater. Today, 2018. 10(Supplement C): p. 12-17.

14. Müller, R., et al., Organic CuTCNQ integrated in complementary metal oxide semiconductor copper back end-of-line for nonvolatile memories. Appl. Phys. Lett., 2006. 89(22): p. 223501.

15. Strukov, D.B., et al., The missing memristor found. Nature, 2008. 453(7191): p. 80-3.

16. Joshua Yang, J., et al., The mechanism of electroforming of metal oxide memristive switches. Nanotechnology, 2009. 20(21): p. 215201.

17. Celano, U., et al., Imaging the Three-Dimensional Conductive Channel in FilamentaryBased Oxide Resistive Switching Memory. Nano Lett, 2015. 15(12): p. 7970-5.

18. Kalantar-zadeh, K., et al., Two dimensional and layered transition metal oxides. Appl. Mater. Today, 2016. 5(Supplement C): p. 73-89.

19. Sawa, A., Resistive switching in transition metal oxides. Mater. Today, 2008. 11(6): p. 28-36.

20. Muenstermann, R., et al., Realization of regular arrays of nanoscale resistive switching blocks in thin films of Nb-doped SrTiO3. Appl. Phys. Lett., 2008. 93(2): p. 023110.

21. Kubicek, M., et al., Uncovering Two Competing Switching Mechanisms for Epitaxial and Ultrathin Strontium Titanate-Based Resistive Switching Bits. ACS Nano, 2015. 9(11): p. 10737-10748. 
22. Messerschmitt, F., et al., Memristor Kinetics and Diffusion Characteristics for Mixed Anionic-Electronic SrTiO3-s Bits: The Memristor-Based Cottrell Analysis Connecting Material to Device Performance. Adv. Func. Mater., 2014. 24(47): p. 7448-7460.

23. Menzel, S., et al., Origin of the Ultra-nonlinear Switching Kinetics in Oxide-Based Resistive Switches. Adv. Func. Mater., 2011. 21(23): p. 4487-4492.

24. Ahmed, T., et al., Transparent Amorphous Strontium Titanate Resistive Memories with Transient Photo-Response. Nanoscale, 2017. 9(38): p. 14690-702.

25. Lee, S.B., et al., Reversible changes between bipolar and unipolar resistance-switching phenomena in a Pt/SrTiOx/Pt cell. Curr. Appl. Phys., 2012. 12(6): p. 1515-1517.

26. Sun, X., et al., Coexistence of the bipolar and unipolar resistive switching behaviours in Au/SrTiO3/Pt cells. J. Phys. D Appl. Phys., 2011. 44(12): p. 125404.

27. Nili, H., et al., Donor-induced performance tuning of amorphous $\mathrm{SrTiO}_{3}$ memristive nanodevices: Multistate resistive switching and mechanical tunability. Adv. Func. Mater., 2015. 25(21): p. 3172-3182.

28. Cai, H.L., X.S. Wu, and J. Gao, Effect of oxygen content on structural and transport properties in $\mathrm{SrTiO}_{3-x}$ thin films. Chem. Phys. Lett., 2009. 467(4-6): p. 313-317.

29. Tonda, S., et al., Synthesis of $\mathrm{Cr}$ and La-codoped $\mathrm{SrTiO}_{3}$ nanoparticles for enhanced photocatalytic performance under sunlight irradiation. Phys. Chem. Chem. Phys., 2014. 16(43): p. 23819-28.

30. Yu, H., et al., Sol-gel hydrothermal synthesis of visible-light-driven Cr-doped SrTiO3 for efficient hydrogen production. J. Mater. Chem., 2011. 21(30): p. 11347.

31. Wang, D., et al., Photophysical and Photocatalytic Properties of $\mathrm{SrTiO}_{3}$ Doped with $\mathrm{Cr}$ Cations on Different Sites. J. Phys. Chemis. B, 2006. 110(32): p. 15824-15830.

32. Funabiki, F., T. Kamiya, and H. Hosono, Doping effects in amorphous oxides. J. Ceram. Soc. Jpn., 2012. 120(11): p. 447-457.

33. Nili, H., et al., Microstructure and dynamics of vacancy-induced nanofilamentary switching network in donor doped $\mathrm{SrTiO}_{3-x}$ memristors. Nanotechnology, 2016. 27(50): p. 505210.

34. Soledade, L.E.B., et al., Room-temperature photoluminescence in amorphous $\mathrm{SrTiO}_{3}$ the influence of acceptor-type dopants. Appl. Phys. A: Mater. Sci. Process., 2002. 75(5): p. 629-632.

35. Goldenberg, E., et al., Effect of $\mathrm{O}_{2} /$ Ar flow ratio and post-deposition annealing on the structural, optical and electrical characteristics of $\mathrm{SrTiO}_{3}$ thin films deposited by $\mathrm{RF}$ sputtering at room temperature. Thin Solid Films, 2015. 590: p. 193-199.

36. Pontes, F.M., et al., Photoluminescence at room temperature in amorphous $\mathrm{SrTiO}_{3}$ thin films obtained by chemical solution deposition. Mater. Chem.Phys., 2003. 77(2): p. 598602.

37. Orhan, E., et al., Origin of photoluminescence in $\mathrm{SrTiO}_{3}$ : a combined experimental and theoretical study. J. Solid State Chem., 2004. 177(11): p. 3879-3885.

38. Yu, H., et al., Elements doping to expand the light response of SrTiO3. J. Photochem. Photobiol. A Chem., 2014. 275: p. 65-71.

39. La Mattina, F., et al., Detection of charge transfer processes in $\mathrm{Cr}$-doped $\mathrm{SrTiO}_{3}$ single crystals. Appl. Phys. Lett., 2008. 93(2): p. 022102.

40. Comes, R.B., et al., Visible light carrier generation in co-doped epitaxial titanate films. Appl. Phys. Lett., 2015. 106(9): p. 092901. 
41. Menke, T., et al., Impact of the electroforming process on the device stability of epitaxial $\mathrm{Fe}$-doped $\mathrm{SrTiO}_{3}$ resistive switching cells. J. Appl. Phys., 2009. 106(11): p. 114507.

42. Linn, E., et al., Complementary resistive switches for passive nanocrossbar memories. Nat. Mater., 2010. 9(5): p. 403-6.

43. $\mathrm{Yu}, \mathrm{S}$., et al., Read/write schemes analysis for novel complementary resistive switches in passive crossbar memory arrays. Nanotechnology, 2010. 21(46): p. 465202.

44. Tappertzhofen, S., et al., Capacity based nondestructive readout for complementary resistive switches. Nanotechnology, 2011. 22(39): p. 395203.

45. Gao, S., et al., Implementation of Complete Boolean Logic Functions in Single Complementary Resistive Switch. Sci. Rep., 2015. 5: p. 15467.

46. Wang, Z.Q., et al. Cycling-induced degradation of metal-oxide resistive switching memory (RRAM). in 2015 IEEE International Electron Devices Meeting (IEDM). 2015.

47. Balatti, S., et al. Pulsed cycling operation and endurance failure of metal-oxide resistive (RRAM). in 2014 IEEE International Electron Devices Meeting. 2014.

48. Kan, D. and Y. Shimakawa, Transient behavior in Pt/Nb-doped SrTiO3 Schottky junctions. Appl. Phys. Lett., 2013. 103(14): p. 142910.

49. Dittmann, R., et al., Scaling Potential of Local Redox Processes in Memristive $\mathrm{SrTiO}_{3}$ Thin-Film Devices. Proc. IEEE, 2012. 100(6): p. 1979-1990.

50. Park, W.Y., et al., A Pt/TiO $/$ Ti Schottky-type selection diode for alleviating the sneak current in resistance switching memory arrays. Nanotechnology, 2010. 21(19): p. 195201.

51. Ahmed, T., et al., Data in Brief, 2018. Submitted.

52. Szot, K., et al., Switching the electrical resistance of individual dislocations in singlecrystalline SrTiO3. Nat. Mater., 2006. 5(4): p. 312-20.

53. Baeumer, C., et al., Quantifying redox-induced Schottky barrier variations in memristive devices via in operando spectromicroscopy with graphene electrodes. Nat. Comm., 2016. 7: p. 12398.

54. Lee, W., et al., Resistance switching behavior of atomic layer deposited $\mathrm{SrTiO}_{3}$ film through possible formation of $\mathrm{Sr}_{2} \mathrm{Ti}_{6} \mathrm{O}_{13}$ or $\mathrm{Sr}_{1} \mathrm{Ti}_{11} \mathrm{O}_{20}$ phases. Sci. Rep., 2016. 6: p. 20550.

55. Lenser, C., et al., Formation and Movement of Cationic Defects During Forming and Resistive Switching in $\mathrm{SrTiO}_{3}$ Thin Film Devices. Adv. Func. Mater., 2015. 25(40): p. 6360-6368.

56. Kwon, J., et al., Oxygen Vacancy Creation, Drift, and Aggregation in TiO2-Based Resistive Switches at Low Temperature and Voltage. Adv. Func. Mater., 2015. 25(19): p. 2876-2883.

57. Meng Lu, Y., et al., Impact of Joule heating on the microstructure of nanoscale TiO2 resistive switching devices. J. Appl. Phys., 2013. 113(16): p. 163703.

58. Li, Y., et al., Nanoscale chemical and valence evolution at the metal/oxide interface: $A$ case study of Ti/SrTiO3. Adv. Mater. Interf., 2016. 3(17): p. 1600201.

59. Stille, S., et al., Detection of filament formation in forming-free resistive switching $\mathrm{SrTiO}_{3}$ devices with Ti top electrodes. Appl. Phys. Lett., 2012. 100(22): p. 223503.

60. Yang, J.J., et al., Diffusion of adhesion layer metals controls nanoscale memristive switching. Adv. Mater., 2010. 22(36): p. 4034-8.

61. Jeong, H.Y., et al., Role of interface reaction on resistive switching of metal/amorphous $\mathrm{TiO}_{2} / \mathrm{Al}$ RRAM devices. J. Electrochem. Soc., 2011. 158(10): p. H979-H982. 
62. Muller, D.A., et al., Atomic-scale imaging of nanoengineered oxygen vacancy profiles in $\mathrm{SrTiO}_{3}$. Nature, 2004. 430(7000): p. 657-661.

63. Sanchez-Santolino, G., et al., Characterization of surface metallic states in $\mathrm{SrTiO}_{3}$ by means of aberration corrected electron microscopy. Ultramicroscopy, 2013. 127: p. 10913.

64. Stoyanov, E., F. Langenhorst, and G. Steinle-Neumann, The effect of valence state and site geometry on $\mathrm{Ti} \mathrm{L}_{3,2}$ and $\mathrm{O} \mathrm{K}$ electron energy-loss spectra of $\mathrm{Ti}_{x} \mathrm{O}_{y}$ phases. Am. Mineral., 2007. 92(4): p. 577-586.

65. Baeumer, C., et al., Verification of redox-processes as switching and retention failure mechanisms in Nb:SrTiO3/metal devices. Nanoscale, 2016. 8(29): p. 13967-75.

66. Meijer, G.I., et al., Valence states of $\mathrm{Cr}$ and the insulator-to-metal transition in $\mathrm{Cr}$ dopedSrTiO3. Phys. Rev. B, 2005. 72(15): p. 155102.

67. Kim, T., et al., Thickness-dependent resistance switching in Cr-doped $\mathrm{SrTiO}_{3}$. J. Korean Phys. Soc., 2012. 61(5): p. 754-758.

68. Janousch, M., et al., Role of Oxygen Vacancies in Cr-Doped $\mathrm{SrTiO}_{3}$ for ResistanceChange Memory. Adv. Mater., 2007. 19(17): p. 2232-2235.

69. Miao, F., et al., Observation of two resistance switching modes in $\mathrm{TiO}_{2}$ memristive devices electroformed at low current. Nanotechnology, 2011. 22(25): p. 254007.

70. Alexandrov, V.E., et al., First-principles study of bulk and surface oxygen vacancies in $\mathrm{SrTiO}_{3}$ crystal. Eur. Phys. J. B, 2009. 72(1): p. 53-57.

71. Cho, E., et al., First-principles study of point defects in rutileTiO2-x. Phys. Rev. B, 2006. 73(19).

72. Tanaka, T., et al., First-principles study on structures and energetics of intrinsic vacancies inSrTiO3. Phys. Rev. B, 2003. 68(20).

73. Andreasson, B.P., et al., Spatial distribution of oxygen vacancies in $\mathrm{Cr}$-doped $\mathrm{SrTiO}_{3}$ during an electric-field-driven insulator-to-metal transition. Appl. Phys. Lett., 2009. 94(1): p. 013513. 
(a)

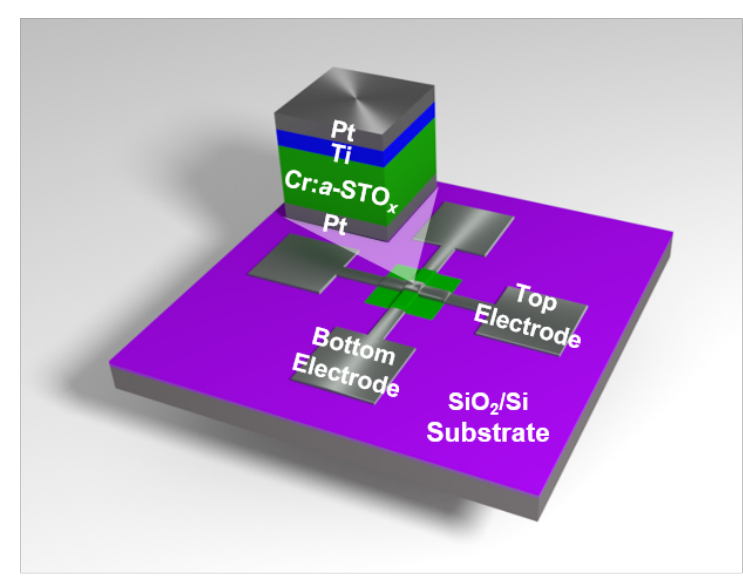

(c)

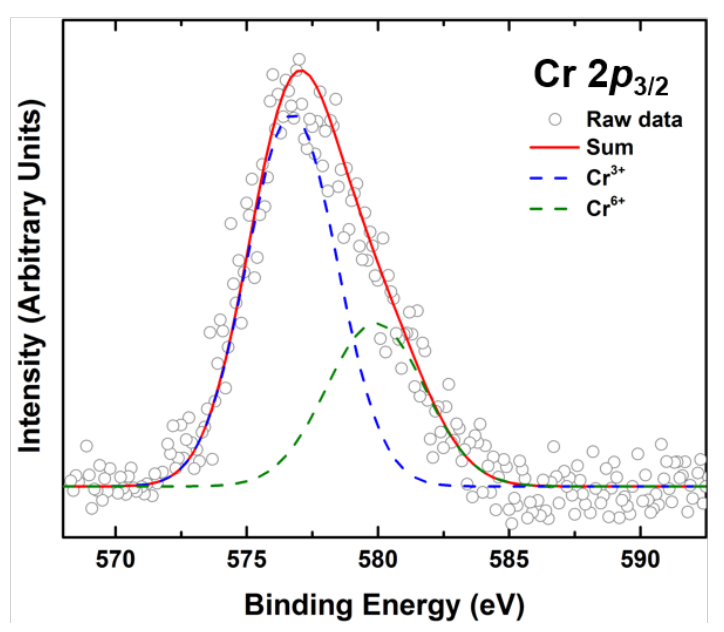

(b)
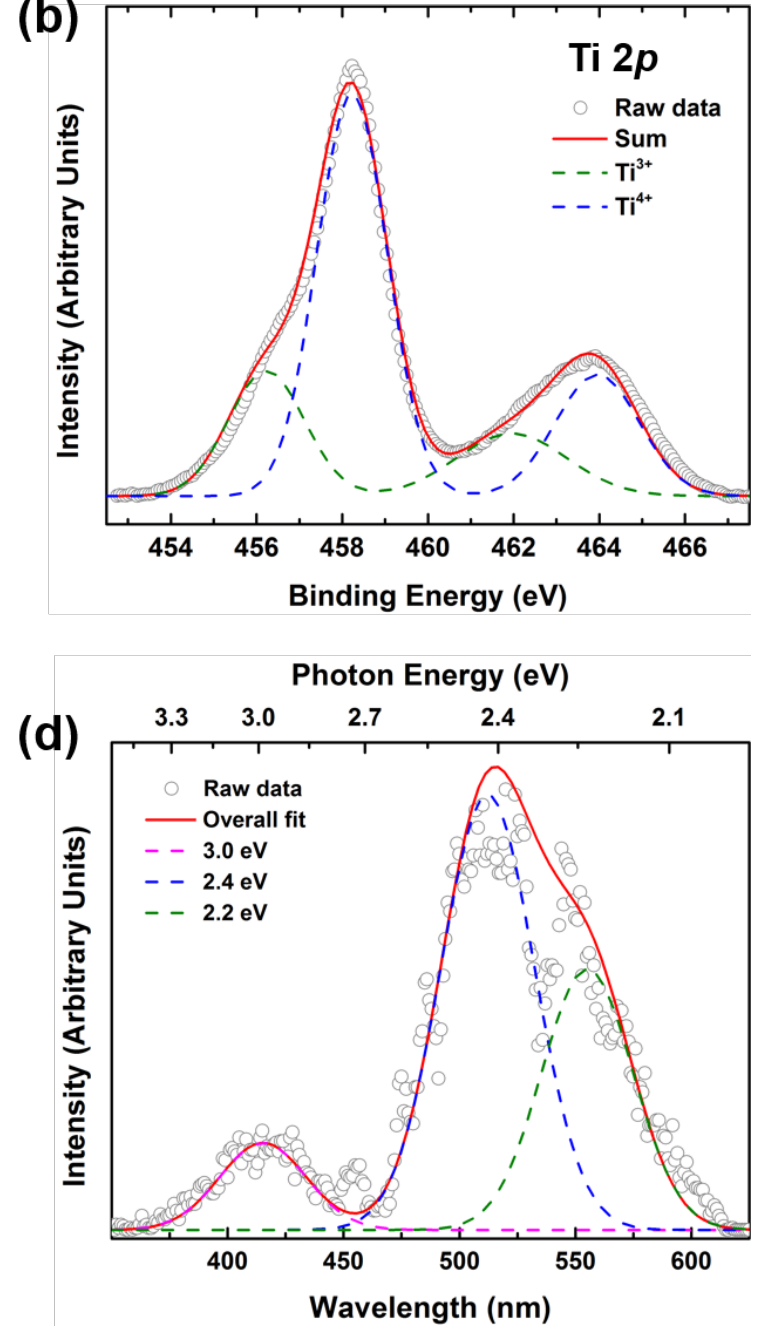

Fig. 1 Device structure and functional oxide characterization. (a) Schematic illustration of a cross-point Cr: $a$-STO $\mathrm{MIM}_{x}$ device. The XPS core-level spectra of (b) Ti $2 p$ and (c) Cr $2 p_{3 / 2}$. (d) Photoluminescence spectra of Cr: $a-\mathrm{STO}_{x}$ collected at an excitation wavelength of $325 \mathrm{~nm}$. 

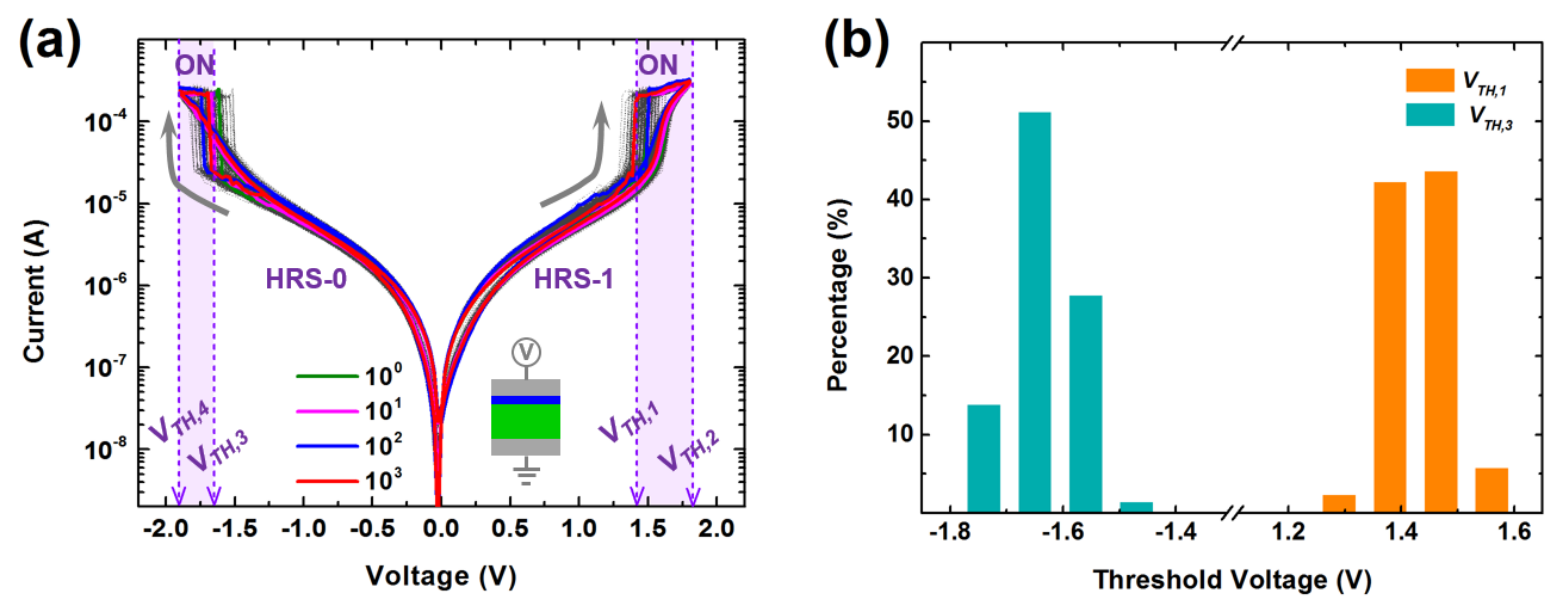

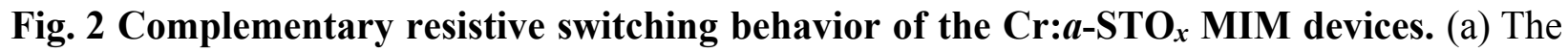
CRS characteristics are repeated for 1000 cycles, while $1^{\text {st }}, 10^{\text {th }}, 100^{\text {th }}$, and $1000^{\text {th }}$ cycles (green, magenta, blue, and red lines, respectively) are highlighted. Inset shows the biasing scheme during CRS switching cycles. (b) Statistic histograms of the CRS threshold voltages, under positive ( $V_{T H, 1}$, orange bars) and negative ( $V_{T H, 3}$, cyan bars) voltages for 1000 cycles. 

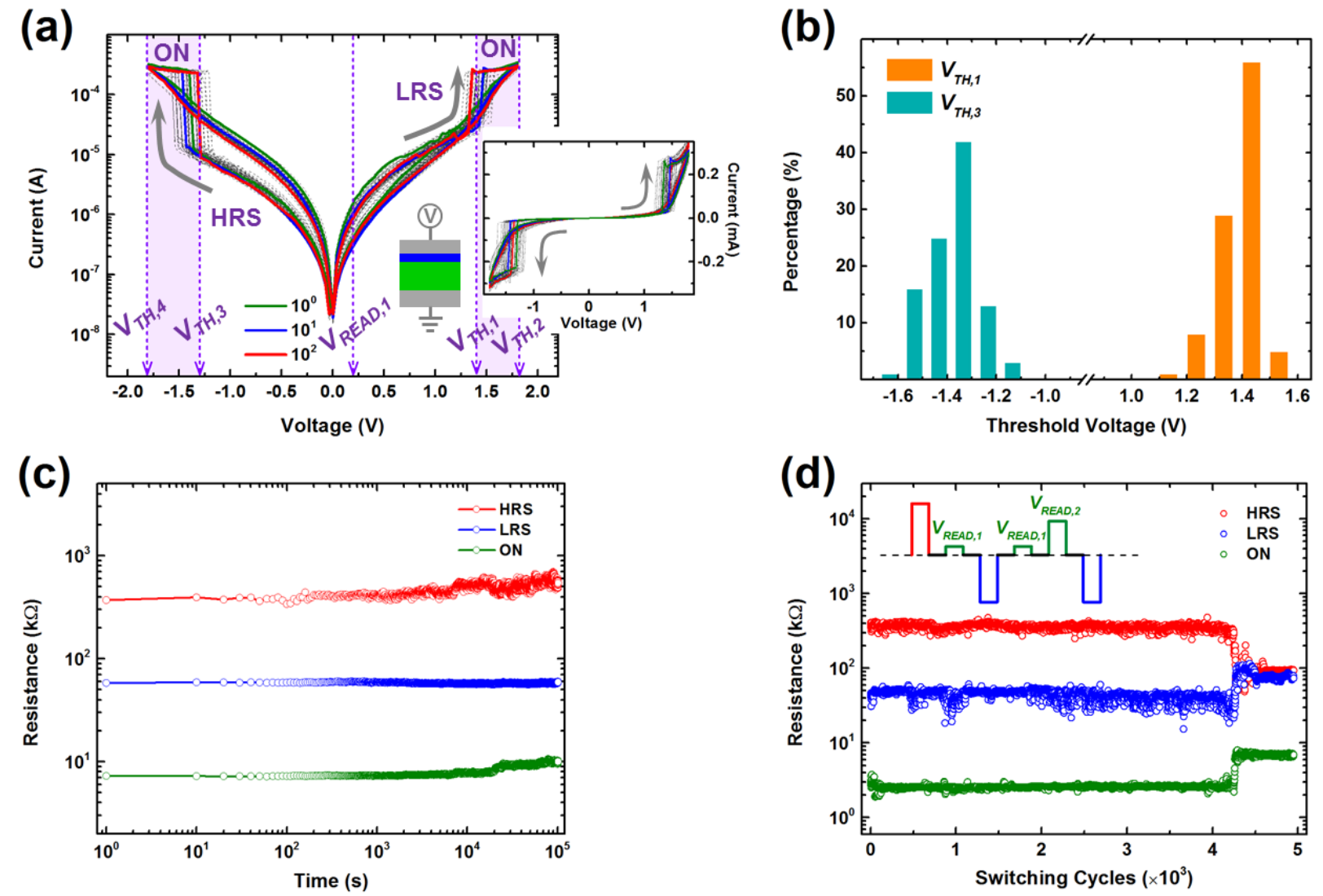

Fig. 3 Peculiar complementary resistive switching of the Cr:a-STO $\mathrm{MIM}^{\mathrm{d}} \mathrm{\text {devices. }}$ (a) Characteristic $I-V$ sweeps for 100 cycles. $1^{\text {st }}, 10^{\text {th }}$, and $100^{\text {th }}$ cycle (green, blue, and red lines, respectively) are highlighted. Inset shows $I-V$ sweeps of 100 cycles on a linear scale along with the bias scheme. (b) Statistic histograms of $V_{T H, 1}$ (orange bars) and $V_{T H, 3}$ (cyan bars) for $100 p$ CRS switching cycles. (c) The retention performance of LRS and HRS measured at constant $V_{R E A D, 1}$ of $+0.2 \mathrm{~V}$ and $\mathrm{ON}$ resistive state measured at constant $V_{R E A D, 2}$ of $+1.6 \mathrm{~V}$ for $10^{5} \mathrm{~s}$. (d) The endurance of $p$-CRS threshold switching for $<5000$ cycles. Inset shows a train of pulses in a complete switching cycle where each pulse has duration of $50 \mu \mathrm{s}$. 
(a)

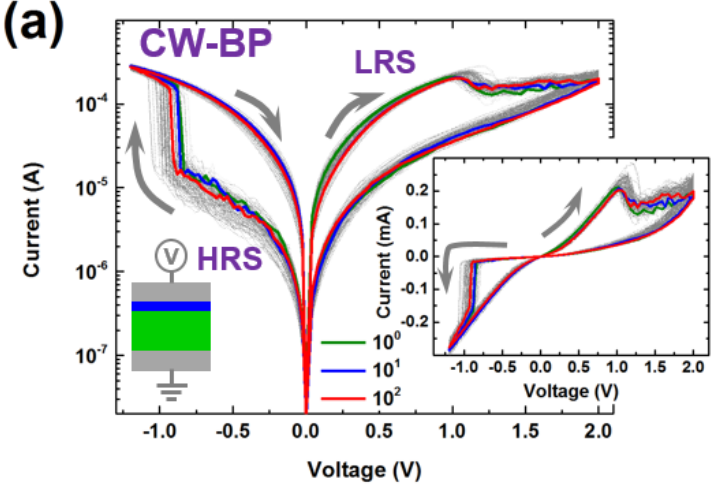

(b)

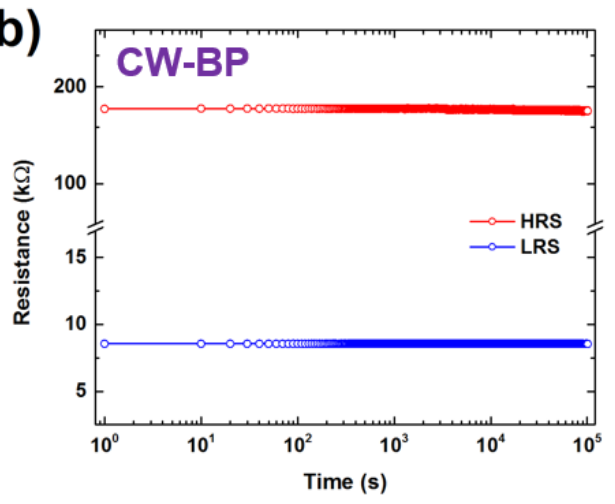

(c)

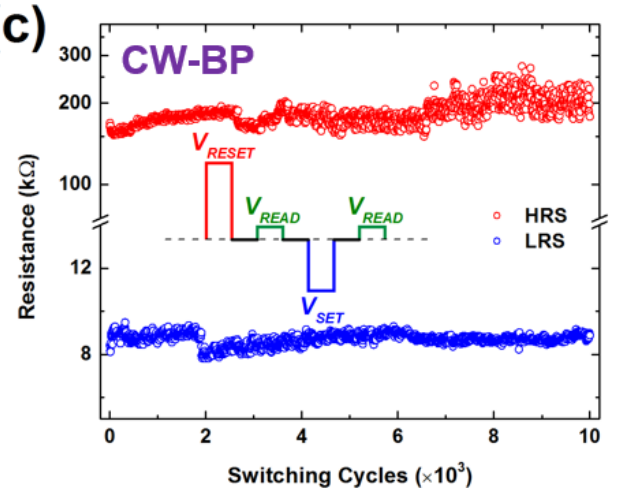

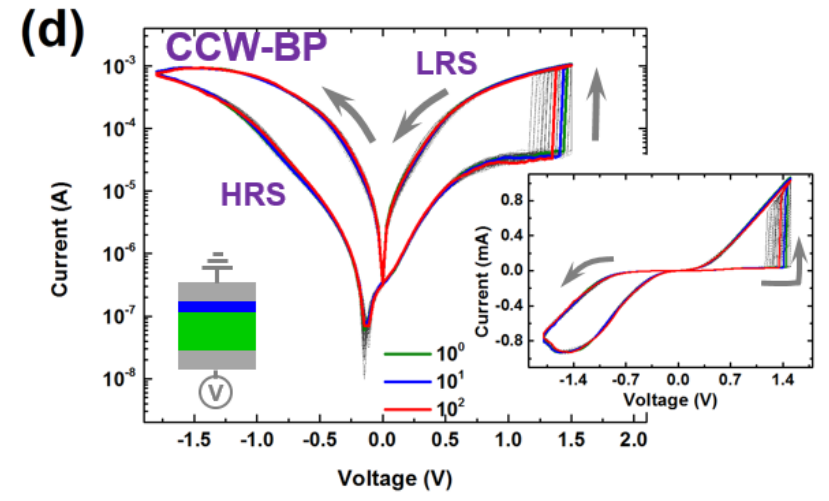

(e)
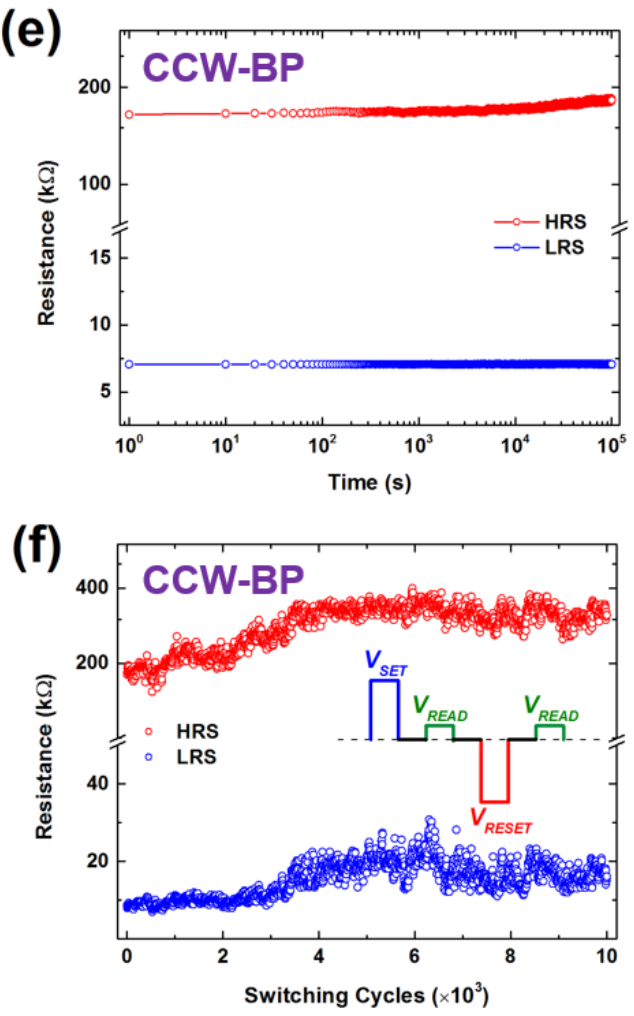

Fig. 4 Electroforming polarity-dependent bipolar resistive switching behavior of the Cr:aSTO $_{x}$ MIM devices. (a) Characteristic $I-V$ sweeps of CW-BP resistive switching for 100 cycles, where the representative $1^{\text {st }}, 10^{\text {th }}$, and $100^{\text {th }}$ cycle (green, blue, and red line, respectively) are highlighted. Inset shows the biasing scheme and $I-V$ sweeps for 100 cycles on a linear scale. (b) Retention performance of CW-BP resistive switching behavior for $10^{5} \mathrm{~s}$ under a constant $V_{R E A D}$ of $+0.2 \mathrm{~V}$. (c) Endurance of CW-BP resistive switching for $10^{4}$ switching cycles. Inset depicts a schematic of a switching cycle where $V_{R E S E T}$ of $+3 \mathrm{~V}, V_{S E T}$ of $-2.7 \mathrm{~V}$ and $V_{R E A D}$ of $+0.2 \mathrm{~V}$ are applied as short pulses each with the duration of $50 \mu \mathrm{s}$. (d) The characteristic $I-V$ sweeps of CCW-BP resistive switching for consecutive 100 cycles, the representative $1^{\text {st }}, 10^{\text {th }}$ and $100^{\text {th }}$ 
cycle (green, blue and red line, respectively) are highlighted. Inset shows the biasing scheme and $I-V$ sweeps for 100 cycles on a linear scale. (e) Retention performance of CCW-BP resistive switching behavior for $10^{5} \mathrm{~s}$ under a constant $V_{R E A D}$ of $+0.2 \mathrm{~V}$ and (f) endurance of CCW-BP resistive switching for $10^{4}$ switching cycles. Inset depicts a schematic of a switching cycle where pulses of $50 \mu \mathrm{s}$ duration (as $V_{S E T}$ of $+2.4 \mathrm{~V}, V_{R E S E T}$ of $-3 \mathrm{~V}$ and $V_{R E A D}$ of $+0.2 \mathrm{~V}$ ) are applied.

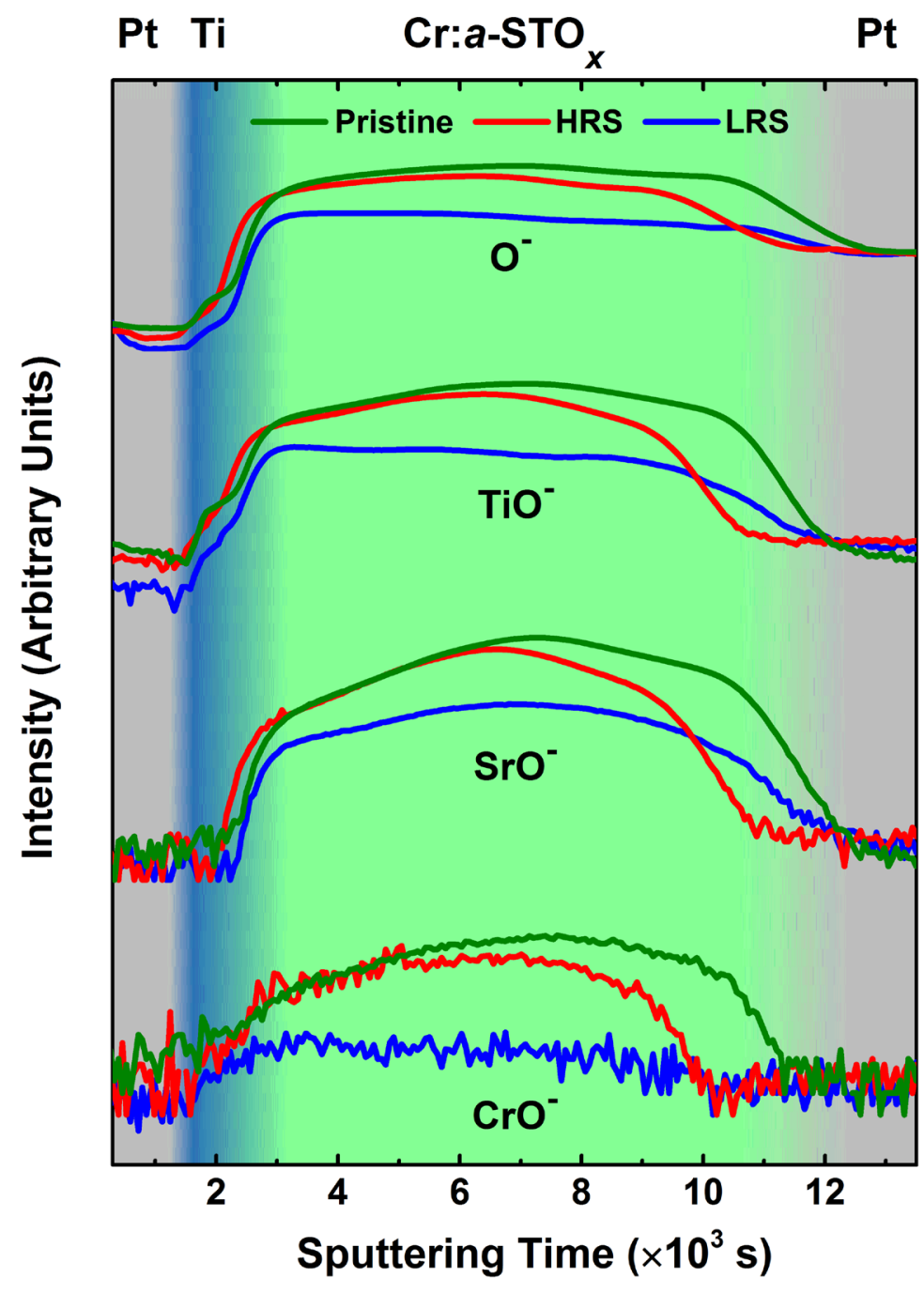

Fig. 5 Nano-SIMS depth profile of the pristine and CW-BP resistive switching Cr:a-STO $x$ MIM devices. The elemental profiles are obtained across the MIM devices in their pristine state (green lines), HRS (red lines), and LRS (blue lines). 

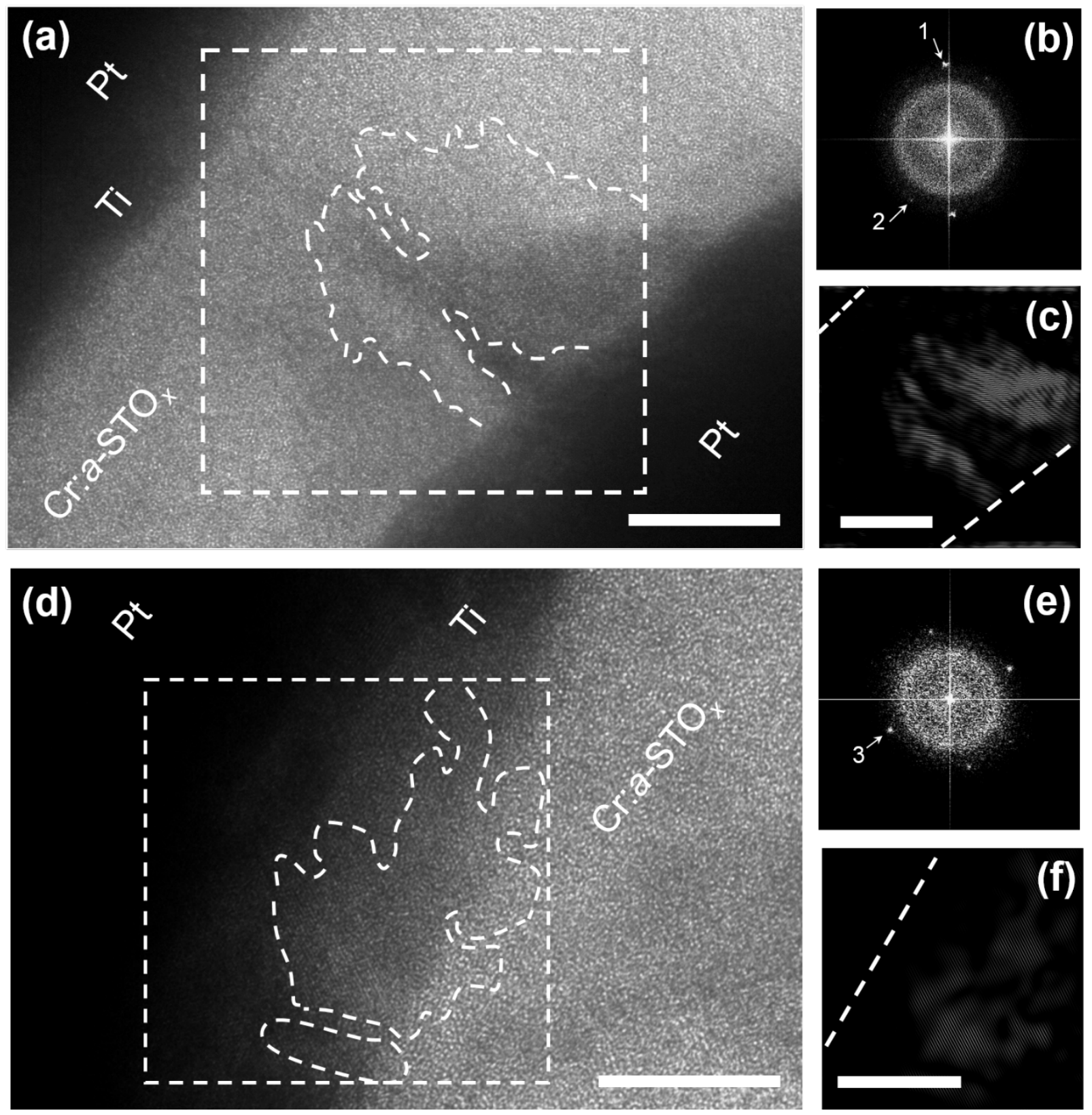

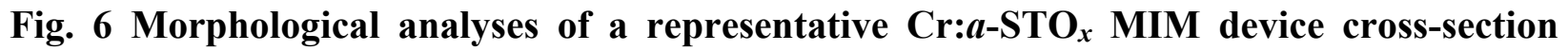
from a device exhibiting CCW-BP resistive switching behavior and pre-set to LRS . (a) TEM micrograph of the MIM device subjected to at least 100 switching cycles and set to LRS prior to the lamella preparation. ROI is enclosed in the box. (b) The FFT diffraction patterns generated from the ROI in (a). (c) The inverse FFT obtained from spot 1 in (b) and highlighted on ROI in (a). (d) TEM micrograph of the top Pt/Ti/Cr: $a-\mathrm{STO}_{x}$ interface with ROI enclosed in 
the box. (e) The FFT diffraction patterns generated from the ROI in (d). (f) The inverse FFT obtained from spot 3 in (e) and highlighted on ROI in (d). All scale bars correspond to $10 \mathrm{~nm}$.

(a)

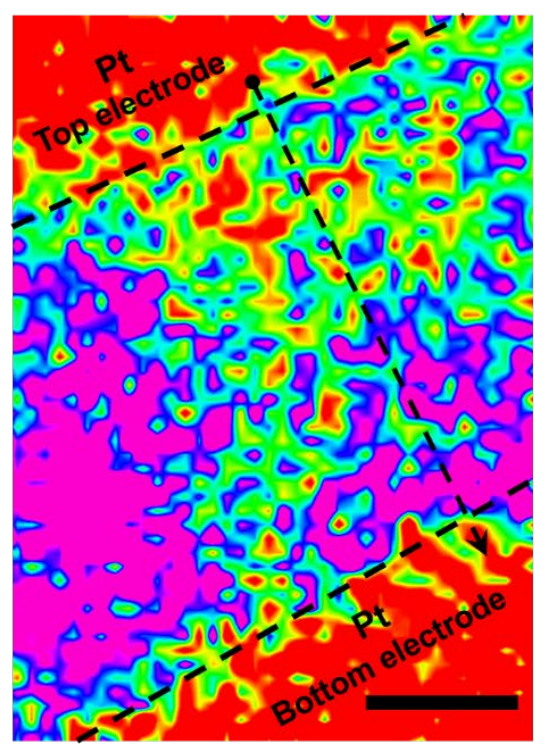

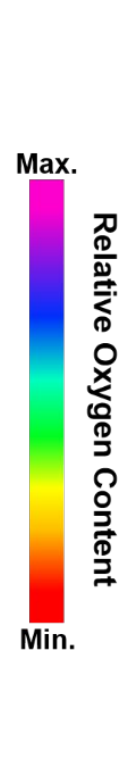

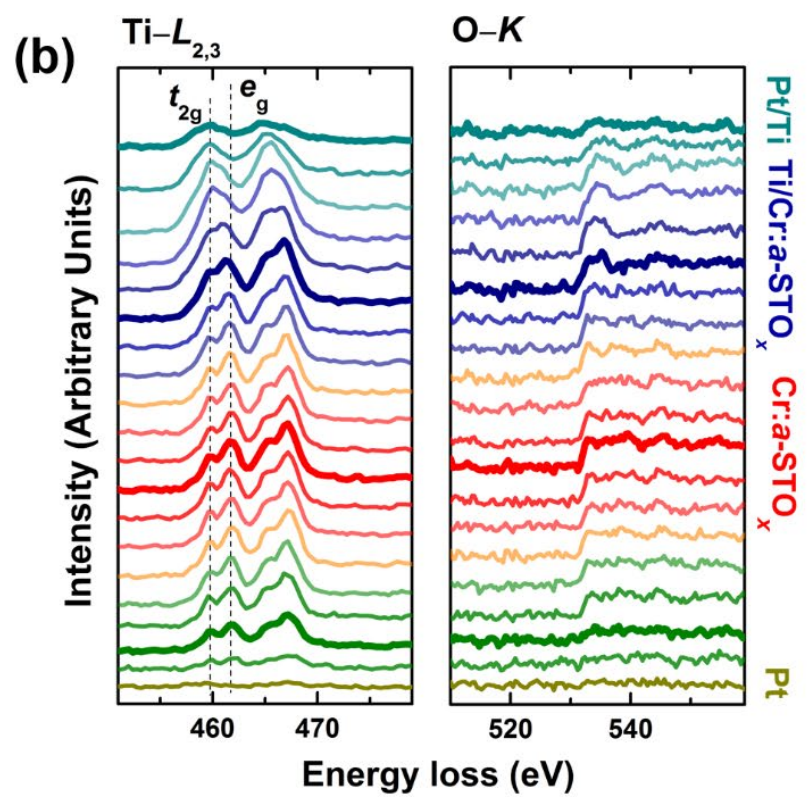

Fig. 7 Electronic structure and compositional analysis of the filamentary path induced by CCW-BP resistive switching. (a) The EELS $O-K$ edge area map of the conductive filament. The area map is collected over the ROI indicated in Fig. 6a. Scale bar: $10 \mathrm{~nm}$. (b) The Ti-L2,3 and $\mathrm{O}-K$ edge profiles along a line scan across the MIM structure, passing over the conductive filamentary path in (a). 


\section{Counter-clockwise bipolar resistive switching}

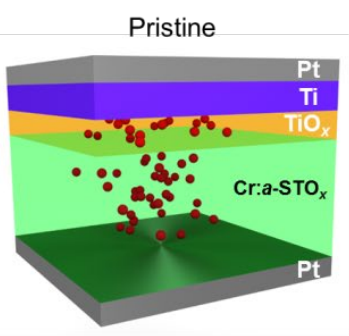

Electroforming/LRS

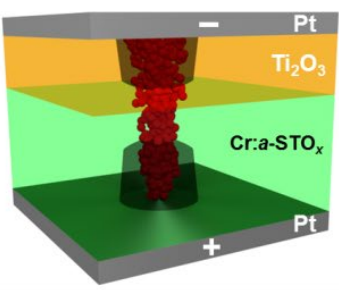

Threshold switching

Electroforming/ON

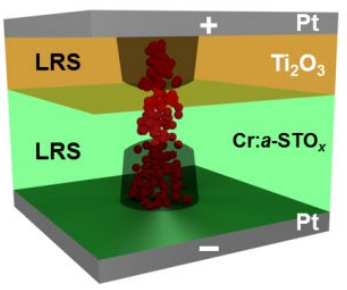

HRS-1

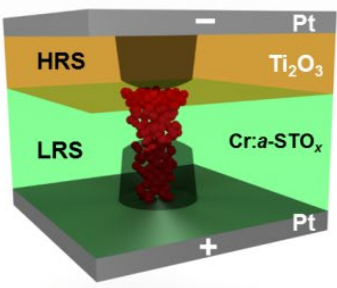

HRS

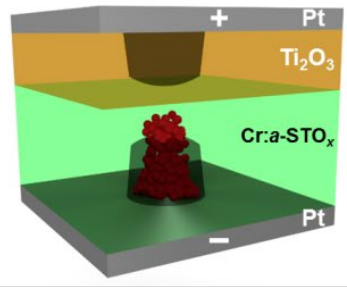

ON

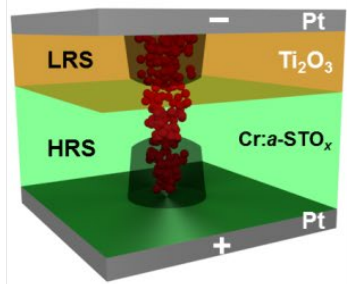

HRS-0

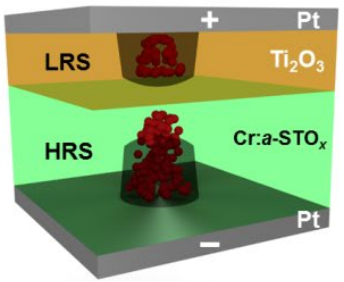

Fig. 8 Schematic illustrations of the possible resistive switching mechanisms induced by variation in the current compliances during initial electroforming. The top panel depicts the changes during and after electroforming in the MIM devices exhibiting CCW-BP switching behavior. The lower panel illustrates the threshold resistive switching mechanism. The red spheres represent the $\mathrm{V}_{\mathrm{oS}}$ and dark structures in the center of schematics represent crystalline regions. 